\title{
Das übersehene Demokratiedefizit \\ Warum die Demokratisierung der EU am europäischen Binnenmarktrecht ansetzen muss
}

DANIEL SEIKEL

Europa erlebt eine schwere Krise. Bei den anstehenden Wahlen zum Europäischen Parlament (EP) im Mai 2019 könnten rechtspopulistische Parteien deutliche Stimmenzuwächse verzeichnen. Die jüngsten Erfolge europakritischer Parteien bei Wahlen und Referenden speisen sich nicht zuletzt aus der Kritik, die Europäische Union (EU) sei undemokratisch und unsozial. Die Demokratisierung der EU steht freilich nicht erst seit dem Erstarken des Rechtspopulismus auf der Agenda fortschrittlicher politischer Kräfte, ist aber heute aktueller und dringender denn je. Jede Strategie, rechtspopulistischen Kräften zu begegnen, muss darauf zielen, die demokratische Qualität europäischen Regierens zu erhöhen. Dazu wurden in der Vergangenheit zahlreiche Vorschläge unterbreitet. Fast alle Reformkonzepte gehen jedoch an einem wesentlichen Demokratieproblem vorbei.

Wenn über das Demokratiedefizit der EU gesprochen wird, stehen zumeist formal-institutionelle Gesichtspunkte im Vordergrund: die eingeschränkten Kompetenzen des EP, die schwache demokratische Legitimation der Europäischen Kommission oder die vergleichsweise starke Stellung sogenannter nicht-majoritärer, technokratischer Institutionen wie der Europäischen Zentralbank. Die meisten der diskutierten Reformvorschläge setzen an solchen formal-institutionellen Aspekten an. Darüber hinaus werden die unvollständigen nicht-formalen Voraussetzungen demokratischer Prozesse problematisiert: die fehlende gemeinsame europäische Identität der EU-Bürger und EU-Bürgerinnen, die mangelnde europäische Öffentlichkeit, das Fehlen echter europäischer Parteien oder die Abwesenheit eines funktionierenden Regierungs-Oppositions-Dualismus.

Ein mindestens ebenso zentrales wie auch strukturelles Demokratieproblem findet hingegen viel $\mathrm{zu}$ wenig Aufmerksamkeit, und wenn doch, dann häufig nur in politik- oder rechtswissenschaftlichen Fachzirkeln. Bei diesem Grundproblem handelt es sich um die Überkonstitutionalisierung des europäischen
Binnenmarktrechts. Die Überkonstitutionalisierung des Binnenmarktrechts ist nicht etwa das Ergebnis eines politisch gesteuerten Prozesses - und damit demokratisch legitimierter Entscheidungen -, sondern die Folge über viele Jahre hinweg kumulierter Rechtschöpfungsakte des Europäischen Gerichtshofes (EuGH). Dies erklärt auch, warum dieses Problem so wenig öffentliche Aufmerksamkeit erfährt. Der Prozess der Überkonstitutionalisierung war gerade nicht Bestandteil öffentlicher politischer Debatten, sondern vollzog sich in Gerichtssälen, überwiegend durch politisch völlig unspektakulär erscheinende Einzelverfahren. Die Überkonstitutionalisierung des Binnenmarktrechts stellt dabei nicht nur ein Demokratieproblem dar, sondern ist überdies auch eine der Ursachen für die soziale Schieflage europäischer Politik. Alle Maßnahmen, die dieses Problem ins Visier nehmen, wären demnach doppelt wirksam: Sie würden nicht nur demokratische Gestaltungsspielräume vergrößern, sondern zugleich institutionelle Hürden für ein sozialeres Europa beseitigen.

\section{Überkonstitutionalisierung als Problem für ein soziales und demokratisches Europa}

Doch worin besteht das Problem des überkonstitutionalisierten Binnenmarktrechts? Das Herzstück des Binnenmarktes sind das Wettbewerbsrecht (Monopol- und Kartellkontrolle, Verbot staatlicher Beihilfen, Vergaberecht) und die vier Grundfreiheiten, die den freien Verkehr von mobilen Produktionsfaktoren - Gütern, Personen, Dienstleistungen und Kapital schützen. Dabei handelt es sich um Instrumente, die von ihrem Wesen her auf Marktschaffung und damit gegen (staatliche) Maßnahmen der Marktbegrenzung ausgerichtet sind. Technisch betrachtet stellen die Grundfreiheiten einen rechtlichen Schutz individueller wirtschaftlicher Freiheiten vor kollektiv-verbindlichen Beschränkungen dar, die aus staatlicher Regel- setzung ebenso hervorgehen können wie aus tarifvertraglichen Vereinbarungen.

Im Prozess der Konstitutionalisierung des Binnenmarktrechts haben die Grundfreiheiten eine rechtlich dominierende Stellung erlangt. Dadurch sind zwei Probleme entstanden: erstens eine faktische Unterordnung sozialer Rechte unter das Primat individueller wirtschaftlicher Freiheiten, und zweitens eine Beschneidung des demokratischen Gestaltungsspielraums.

Die daraus resultierenden Konsequenzen für eine Marktergebnisse korrigierende und damit Wirtschaftsfreiheiten einschränkende Politik sind unmittelbar ersichtlich: Ihr sind deutliche europarechtliche Grenzen gesetzt. Das betrifft zum einen legislative Maßnahmen, sowohl auf europäischer wie auf nationaler Ebene, etwa bei gesetzlichen Maßnahmen zur Regulierung transnationaler Lohnkonkurrenz. Der vom EuGH überwachte Grundfreiheitenschutz hat nicht nur die Spielräume nationaler Gesetzgeber eingeschränkt, wie die Revision der Entsenderichtlinie zeigt: Hier musste auch der europäische Gesetzgeber sehr darauf achten, nicht die Dienstleistungsfreiheit zu stark einzuschränken. Polen und Ungarn, vereint in ihrer Gegnerschaft zur Regulierung der Arbeitnehmerentsendung, bezweifeln, dass dies gelungen sei, und haben jüngst die Revision vor dem EuGH angefochten. Aber auch tarifvertragliche Regelungen sowie Arbeitskampfmaßnahmen, mit denen Tarifverträge erkämpft werden sollen, werden vom Grundfreiheitenschutz beeinträchtigt. In der Vergangenheit sind dadurch die Tarifautonomie und das Streikrecht immer wieder eingeschränkt worden. Ein weiteres Beispiel ist die Unternehmensmitbestimmung. Sie ist zwar vom EuGH in der Erzberger-Entscheidung für grundfreiheitenfest erklärt worden. ${ }^{1}$

1 In der Erzberger-Entscheidung (C-566/14 vom 18. 07. 2017) hatte der EuGH zu befinden, ob die deutsche Unternehmensmitbestimmung gegen 
Jedoch wird sie von der aus der Personenfreizügigkeit hervorgehenden Niederlassungsfreiheit systematisch unterlaufen. Das vom EuGH zunehmend expansiv ausgelegte Recht von Unternehmen, ihren Sitz frei von nationalen Bestimmungen von Land zu Land verlagern und damit zwischen den verschiedenen nationalen Rechtsformen wählen zu dürfen, droht die Unternehmensmitbestimmung auszuhebeln (vgl. dazu auch Hassel/von Verschuer in diesem Heft). Dabei muss die Umgehung der Mitbestimmung gar nicht das Hauptmotiv von Unternehmen sein; vielmehr ist sie häufig ein „Kollateralschaden" des primärrechtlich verbrieften Rechts von Unternehmen, den Verwaltungssitz zu verlegen, um, beispielsweise, möglichst wenig Steuern zu zahlen.

Aber warum ist die hervorgehobene Stellung der Grundfreiheiten auch ein Demokratieproblem? Dies hat der ehemalige Richter am Bundesverfassungsgericht, Dieter Grimm, besonders deutlich veranschaulicht. ${ }^{2}$ Das europäische Verfassungsrecht ist mit starken ordnungspolitischen Weichenstellungen aufgeladen, die im Ergebnis eine dem nationalen Recht übergeordnete marktliberale Wirtschaftsordnung konstitutionalisieren. Derartig starke ordnungspolitische Weichenstellungen jedoch sind dem nationalen (Verfassungs-)Recht normalerweise fremd. So steht etwa das deutsche Grundgesetz den Grundzügen der Wirtschaftsordnung weitaus „offener“ gegenüber, als dies bei der europäischen Wirtschaftsverfassung der Fall ist. Generell gilt, je mehr und je detaillierter verfassungsrechtlich vorab festgelegt ist, desto weniger Gestaltungsspielraum bleibt für die Politik. Durch die ordnungspolitisch ungewöhnlich starren konstitutionellen Weichenstellungen auf der europäischen Ebene wird also die Gestaltungsfreiheit demokratischer Politik sowohl auf nationaler als auch europäischer Ebene eingeengt. Der demokratische Wettbewerb um die „richtige“ Wirtschaftsordnung ist rechtlich eingeschränkt. Die starke Stellung des Binnenmarktrechts hat zur Folge, dass politische Auseinandersetzungen zwischen Befürwortern und Gegnern einer stärkeren Regulierung von Märkten nicht unter rechtlicher „Waffengleichheit" stattfinden; das Binnenmarktrecht ist eine starke Machtressource in den Händen der Gegner von politischen Eingriffen in Marktprozesse. Da so bestimmte politische Programmatiken - vor allem wirtschaftsliberale - bevorzugt werden, während andere - wie etwa das Programm eines regulierten Kapitalismus - systematisch benachteiligt werden, ist die Korrektur der Überkonstitutionalisierung des Binnenmarktrechts eine zentrale Herausforderung gerade auch für Gewerkschaften.

\section{Viele Vorschläge zur Demokratisierung der EU laufen ins Leere}

Angesichts dieser massiven Beschneidung demokratischer Gestaltungsfreiheit kratzen viele der derzeit diskutierten Vorschläge zur Behebung des Demokratiedefizits europäischen Regierens bestenfalls an der Oberfläche des Problems oder laufen gar gänzlich ins Leere. Dies gilt für den geforderten Spitzenkandidaten-Automatismus bei der Ernennung des Kommissionspräsidenten genauso wie für die Forderungen nach einem europäischen Finanzminister oder transnationalen Kandidatenlisten für die Europawahlen. Selbst die grundsätzliche und wichtige Forderung nach einer institutionellen Aufwertung des Europäischen Parlaments im europäischen Institutionengefüge hätte nicht den Effekt, den es haben könnte, solange die dort verhandelten Materien unter einem derartigen Grundfreiheitenvorbehalt verbleiben. Folglich sollten die politischen Anstrengungen besser auf eine Befreiung europäischer und nationaler Politik aus den Fesseln der marktliberalen Bestandteile des europäischen Binnenmarktes konzentriert werden als auf so manches andere, das derzeit diskutiert wird von als Meilensteinen der Demokratisierung verkauften Marketingmaßnahmen wie der Online-Befragung zur Abschaffung der Zeitumstellung ganz zu schweigen.

\section{Das Primat des Politischen über den Binnenmarkt herstellen}

Wie ließe sich das Primat des Politischen über das Binnenmarktrecht wiederherstellen? Hier$\mathrm{zu}$ sind drei Maßnahmen denkbar: Erstens, die umfassendste und wohl auch wirksamste Option wäre ein konstitutioneller Neustart der EU. Kern dieses Vorschlages ist eine „echte" europäische Verfassung, die nur diejenigen Elemente beinhaltet, die typsicherweise in

die europarechtlich geschützte Arbeitnehmerfreizügigkeit verstoße. Die Richter verneinten dies (siehe https://www.boeckler.de/wsi_110074. htm).

2 Grimm, D. (2016): Europa ja - aber welches? Zur Verfassung der europäischen Demokratie, München.
Verfassungen enthalten sind, also Regelungen über Kompetenzen, Organe, Verfahrensweisen sowie politische und soziale Grundrechte. Alle anderen Elemente, darunter auch das Binnenmarktrecht, würden indessen in das „einfache" EU-Recht, das sogenannte Sekundärrecht, überführt. Dadurch würde die europäische Politik die „Oberhand“ über die Grundfreiheiten erlangen, da diese als Rechtsbestände ohne Verfassungsrang politisch leichter korrigiert werden könnten. Das Ergebnis wäre eine insofern „offenere“ europäische Verfassung, als sie gegenüber verschiedenen ordnungspolitischen Leitbildern offener wäre als die derzeitige Wirtschaftsverfassung. Zweitens, die europäischen Verträge könnten - wie vom Europäischen Gewerkschaftsbund vorgeschlagen - um ein Soziales Fortschrittsprotokoll ergänzt werden, das sozialen Rechten einen grundsätzlichen Vorrang vor den Grundfreiheiten einräumen würde. Schließlich, drittens, könnten Ausnahmebereiche definiert werden, für die die Grundfreiheiten und das Wettbewerbsrecht nicht gelten, vorzugsweise im Vertragsrecht verankert, möglicherweise aber auch im Sekundärrecht. Die so festgelegten Ausnahmebereiche würden die Tarifautonomie, Tarifverträge und das Streikrecht aus dem Anwendungsbereich der Grundfreiheiten und des Wettbewerbsrechts herausnehmen.

Die zuletzt immer lauter gewordene Kritik an Europa bezieht sich in der Regel auf zwei Defizite: Erstens sei die EU nicht demokratisch genug; zweitens sei die EU nicht sozial genug. Diese Kritik sollte ernst genommen werden. Beide Defizite sind viel enger miteinander verzahnt, als man vielleicht meinen könnte, und gehen auf dieselbe Ursache zurück: Die Überkonstitutionalisierung des Binnenmarktrechts. Die dominante Stellung der Grundfreiheiten ist gewiss nicht die einzige Ursache dieser Probleme; hier ist insbesondere die neue europäische Economic Governance mit all ihren negativen Auswirkungen auf Demokratie, Wohlfahrtsstaaten und soziale Rechte zu nennen. Dennoch muss jede Strategie zur Demokratisierung der EU am europäischen Binnenmarktecht ansetzen.

\section{AUTOR}

DANIEL SEIKEL, Dr., ist Wissenschaftler am Wirtschafts- und Sozialwissenschaftlichen Institut (WSI) der Hans-Böckler-Stiftung. Arbeitsschwerpunkt: Europäische Politik.

daniel-seikel@boeckler.de 


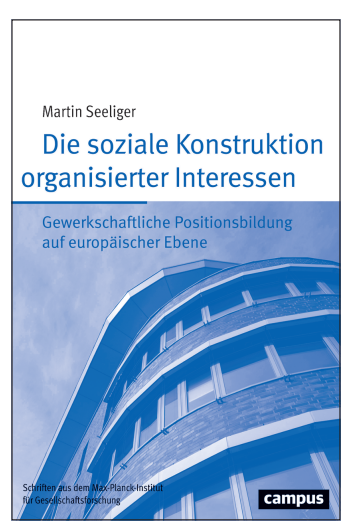

MARTIN SEELIGER DIE SOZIALE KONSTRUKTION ORGANISIERTER INTERESSEN GEWERKSCHAFTLICHE POSITIONSBILDUNG AUF EUROPÄISCHER EBENE

Campus Verlag, Frankfurt a. M./New York 2017

ISBN 978-3-593-50786-6 304 Seiten, 39,95€
W

ährend sowohl unter Praktikern als auch in der Literatur häufig die Notwendigkeit gewerkschaftlicher Kooperation in Europa betont wird, besteht Uneinigkeit über deren Erfolg. Martin Seeliger macht in der sozialwissenschaftlichen Debatte zwei Strömungen aus. Aus einer optimistischen Sicht würden eher normative Empfehlungen geäußert und eine stärker auf europäische Abstimmungsprozesse ausgerichtete Gewerkschaftspolitik auch für möglich gehalten. Demgegenüber erscheine aus pessimistischer Sicht die gewerkschaftliche Positionsbildung auf europäischer Ebene vor dem Hintergrund der Heterogenität der Mitgliedstaaten sowie der Gewerkschaften selbst sehr unwahrscheinlich. Seeliger legt seiner Untersuchung den aus dieser Sicht anti-intuitiven Befund zugrunde, dass in den wichtigsten Feldern der europäischen Tarifpolitik dennoch gemeinsame Positionen der europäischen Gewerkschaften etabliert werden konnten, und geht der Frage nach, wie dies möglich war. Dazu beleuchtet er die gewerkschaftliche Positionsbildung: zum einen in Bezug auf zwei europäische Gesetzgebungsverfahren, die Dienstleistungsrichtlinie und die Durchsetzungsrichtlinie zur Entsenderichtlinie, zum zweiten blickt er auf die Debatte zu einem europäischen Mindestlohn sowie die gewerkschaftliche Lohnkoordinierung in der Eurozone.

Seeligers Buch liegt eine breite empirische Basis zugrunde. Neben der Analyse relevanter Dokumente führte er 87 Interviews mit Vertretern europäischer und nationaler Gewerkschaften. In den Fällen der Dienstleistungsrichtlinie, der Durchsetzungsrichtlinie und des Mindestlohns betrachtet er je drei Gewerkschaftsföderationen aus Polen, Ungarn und Schweden sowie auf europäischer Ebene den Europäischen Gewerkschaftsbund (EGB) und vier Branchenverbände (insbesondere die Europäische Bau- und Holzarbeiterföderation, EFBH). Die Auswahl berücksichtigt die in diesen Bereichen besonders virulenten Unterschiede zwischen Ost- und Westeuropa in Bezug auf Arbeits- und Sozialstandards und die Machtressourcen der Gewerkschaften (S. 23). Für das Fallbeispiel der Koordinierung der Tarifpolitik konzentriert Seeliger sich auf den europäischen Branchengewerkschaftsverband IndustriAll sowie die deutsche IG Metall, da der (deutschen) Metallindustrie für die Wirtschaft der Eurozone besondere Bedeutung zukomme und die tarifpolitischen Koordinierungsinitiativen in diesem Bereich am weitesten fortgeschritten sind (S. 23). Die zunächst etwas unsystematisch anmutende Auswahl der Untersuchungsländer und -gewerkschaften lässt sich also mit ihrer speziellen Relevanz in den betrachteten Bereichen begründen, die Seeliger als Aspekte „des größeren Ganzen“ der europäischen Tarifpolitik analysiert (S.96).

Dazu zieht er verschiedene theoretische Ansätze heran. Seeliger strebt an, Elemente der vorwiegend pragmatistischen Handlungs- und Sozialisationstheorie und der Organisationssoziologie $\mathrm{zu}$ einem Modell zu integrieren (S.101). Sein Ausgangspunkt ist die pragmatistische
Handlungstheorie, die von der grundsätzlichen Kompromissneigung von Menschen und einer Ergebnisoffenheit des Handelns ausgeht und eine akteurszentrierte und prozessuale Perspektive auf Handlungsverläufe einnimmt (S. 127). Im Gegensatz zu der von Seeliger „pessimistisch“ genannten Sicht auf gewerkschaftliche Kooperation in Europa werden aus dieser Perspektive Interessen als sozial konstruiert und scheinbar „objektive“ Interessengegensätze als überwindbar verstanden (S. 41). Indem er Konzepte aus der Organisationstheorie hinzuzieht, wie das der „Entkopplung“ von Umwelterwartungen und organisationaler Realität (Meyer/Rowan $1977^{1}$ ), will Seeliger aber die Möglichkeit in die Betrachtung einbeziehen, dass gemeinsame Positionen zwar etabliert werden, ohne dass aber die realen Interessengegensätze der Akteure aufgelöst würden (S.128). Auch diese Konzepte aus der Organisationstheorie werden von Seeliger um weitere Überlegungen ergänzt. Das Auseinanderfallen von „Reden“ und „Handeln“ könne nicht nur der Befriedigung aktueller Erwartungen zur Legitimitätssicherung dienen (wie bei Meyer/Rowan 1977). Vielmehr ließe sich mit den Konzepten der „imagined $f u$ tures" und "politics of expectations" von Beckert $(2016)^{2}$ der Aspekt fassen, dass Zukunftserwartungen für das Handeln von Akteuren in der Gegenwart strukturierende Wirkung entfalten können. Zukunftshoffnungen seien in der Geschichte der Arbeiterbewegung ein entscheidendes Instrument politischer Mobilisierung gewesen (S.122f.).

Mit diesem theoretischen Werkzeugkasten begibt sich Seeliger in die empirische Analyse. Im Politikfeld der Dienstleistungsfreiheit ist die polit-ökonomische $\mathrm{He}$ terogenität der EU-Mitgliedsländer eine entscheidende Hintergrundbedingung für die gewerkschaftliche Positionsbildung. Der ursprüngliche Vorschlag für die Dienstleistungsrichtlinie bedeutete aus Sicht westeuropäischer Gewerkschaftsvertreter eine Gefahr für westeuropäische Arbeits- und Sozialstandards, wurde von osteuropäischen Gewerkschaftsvertretern jedoch zunächst begrüßt. Im Laufe des Diskussionsprozesses konnte aber eine Einigung unter dem Begriff „Soziales Europa“ erreicht werden. Dieses Zukunftsversprechen (aus Sicht der Osteuropäer) verbunden mit einer Machtasymmetrie zwischen west- und osteuropäischen Gewerkschaftsvertretern, die die Durchsetzungsfähigkeit Letzterer auf europäischer Ebene beeinträchtigt, ermöglichte laut Seeligers Analyse die gemeinsame Position in diesem Politikfeld (S.163). Das Konzept eines „Sozialen Europas“ fungiert hier als identitätsstiftender Bezugsrahmen, der die anhaltende Mobilisierung kollektiven Handelns ermöglicht (S. 44).

Meyer, J.W./ Rowan, B. (1977): Institutionalized Organizations: Formal Structure as Myth and Ceremony, in: American Journal of Sociology 83 (2), S. 340-363.

2 Beckert, J. (2016): Imagined Futures: Fictional Expectations and Capitalist Dynamics, Cambridge, MA 
Im Fall der Durchsetzungsrichtlinie zur Entsenderichtlinie kam zu der Konfliktlinie zwischen Ost- und Westeuropa eine zweite Dimension des Konflikts zwischen dem EGB auf der einen und den europäischen Branchengewerkschaften (insb. der EFBH) sowie zahlreichen nationalen Mitgliedsorganisationen auf der anderen Seite hinzu. Seitens der Branchenverbände wird die integrationistische Haltung des EGB (die u. a. durch die externe Bezuschussung des EGB durch die Europäische Kommission erklärt werden könne) angeprangert (S. 64), während der EGB seinerseits die Kurzsichtigkeit der Branchenverbände kritisierte, die das langfristige Ziel einer Stärkung der europäischen Regulierungsebene und tarifpolitischen Arena nicht im Blick hätten (S. 181). Seeliger macht hier mit Verweis auf Martin Höpner - ein interessantes „Sozialisationsparadox“ im Mehrebenensystem europäischer Gewerkschaftspolitik aus: gerade die integrationistische Sozialisation der EGB-Funktionäre wirke als Integrationshemmnis, wenn aufgrund dessen keine gemeinsame gewerkschaftliche Position etabliert werden könne (S. 250).

Eine andere Konfliktkonstellation zeigt sich im Falle der Diskussionen um einen europäischen Mindestlohn. Dieser wird insbesondere von den schwedischen Gewerkschaften abgelehnt, da eine Gefährdung der Tarifautonomie durch staatliche Eingriffe befürchtet wird. Zwar konnte unter dem Dach des EGB eine nominelle Einigung auf eine Kampagne zum europäischen Mindestlohn erzielt werden, diese wurde allerdings nie umgesetzt (S. 81).

Im Feld der Lohnpolitik schließlich stellt Seeliger die Frage, warum die Gewerkschaften an ihren Initiativen zur Lohnkoordinierung festhalten, obwohl bisher die Ziele - Verhinderung eines Unterbietungswettbewerbs und Gewährleistung makroökonomischer Stabilität - nicht erreicht worden seien (S.89). In diesem Untersuchungsfeld betrachtet Seeliger insbesondere die tarifpolitischen Strategien der IG Metall. Obgleich sich die IG Metall zur Lohnkoordinierungsregel bekenne, weiche die tatsächliche Lohnentwicklung in der Metall- und Elektroindustrie im Untersuchungszeitraum von 1998 bis 2013 fast durchgehend vom Koordinierungsziel ab, dass hier anhand des Produktivitätszuwachses der deutschen Metallindustrie und alternativ der deutschen Inflationsrate oder der Zielinflationsrate der EZB berechnet wird. Die Verweise der IG Metall-Vertreter auf die ungünstigen Rahmenbedingungen für Tarifpolitik in Deutschland in den letzten Jahren und Unterschiede zwischen Tarif- und Effektivlohnentwicklung werden von Seeliger angeführt, jedoch nicht weiter vertieft. Es geht ihm vielmehr um die Frage, ob man die Lohnkoordinierung nun aus organisationstheoretischer Sicht als einen Fall der „Entkopplung“ ansehen müsse. Dies ist aus Seeligers Sicht nicht der Fall. Vielmehr sei die zeitliche Dimension von Bedeutung, denn die Initiative bezöge einen wichtigen Teil ihrer Legitimität in der Gegenwart daraus, dass sie die Perspektive der Etablierung einer europäischen Verhandlungsarena in der Zukunft beinhalte (S. 218f.). Mit Beckerts Ansatz der fiktionalen Er- wartungen ließe sich - ähnlich wie bei der Zielvorstellung eines „Sozialen Europa“ - die Proklamation, dass Lohnkoordinierung prinzipiell möglich sei, als ein wichtiges strategisches Mobilisierungselement interpretieren, um sie in Zukunft tatsächlich erreichen zu können (S. 233).

Mit seiner Studie will Seeliger die Lücke schließen, die seines Erachtens darin besteht, dass die sozialwissenschaftliche Auseinandersetzung bisher noch keine systematische Analyse gewerkschaftlicher Positionsbildung hervorgebracht habe (S. 71). Um dies zu erreichen, werden verschiedene Theorieansätze produktiv miteinander verknüpft, die verschiedene Aspekte der ihrerseits vielseitigen empirischen Fälle unterschiedlich gut erklären können. In allen Fällen scheinen die Ergebnisse der Aushandlungsprozesse maßgeblich von Machtrelationen zwischen den Akteuren beeinflusst zu sein, u.a. der unterschiedlichen Position der Gewerkschaften innerhalb der nationalen Systeme der Arbeitsbeziehungen, damit zusammenhängend unterschiedlichen Notwendigkeiten und Möglichkeiten der Durchsetzung im europäischen Rahmen, oder auch der Angewiesenheit auf externe Ressourcen im Falle des EGB. Seeliger selbst weist die Kritik am pragmatistischen Ansatz, dass dieser Machtverhältnisse zwischen Akteuren zu wenig berücksichtige, zurück (S.106f.). Machtverhältnisse würden nicht ausgeblendet, sondern als „Produkte wechselseitig aufeinander bezogener Sinnhorizonte rekonstruiert" (S. 107). Andererseits wird zum Ende des Buches auf die Verschiebung der Machtverhältnisse im Prozess der europäischen Integration zuungunsten der Gewerkschaften hingewiesen (S.251) und beispielsweise gefragt, inwieweit sich eine koordinierte Tarifpolitik - sofern sie tatsächlich gewollt sei - von der IG Metall überhaupt gegenüber der Kapitalseite durchsetzen ließe (S. 252). Es wird damit auf soziale Bedingungen verwiesen, die außerhalb der konkreten Interaktion der Gewerkschaftsvertreter liegen. Wenn man davon ausgeht, dass die gewerkschaftliche Positionsbildung sich immer schon vor dem Hintergrund dieser Machtverhältnisse vollzieht und die Standpunkte der beteiligten Akteure durch ihre Position innerhalb dieses Gefüges bereits geprägt sind, könnte ein theoretischer Ansatz, der dies noch stärker berücksichtigt, eventuell fruchtbar für die weitere Untersuchung gewerkschaftlicher Positionsbildung in Europa sein.

Seeligers fundierte und sehr gut zu lesende Analyse regt dazu an, den „Erfolg“ oder „Misserfolg“ gewerkschaftlichen Handelns in Europa nicht nur an ökonomischen Indikatoren oder konkreten Regulierungsergebnissen zu messen, sondern die Aushandlungsprozesse zwischen europäischen Gewerkschaften sowie die Bedingungen, unter denen sie zustande kommen, selbst als wichtigen Forschungsgegenstand zu betrachten.

NELE DITTMAR, BERLIN 


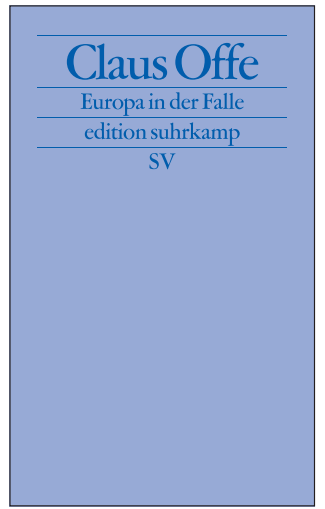

OFFE, CLAUS EUROPA IN DER FALLE

Suhrkamp

Berlin, 2016

ISBN 978-3-518-12691-2 160 Seiten, $16,-€$
$\mathrm{Z}$ ur Krise der Europäischen Union (EU) und ihrer Währungsunion gibt es eine stetig anwachsende Fülle kritischer Fach-Publikationen. Umso wichtiger sind gut informierte und dennoch leicht zu lesende Bücher, die auf knappem Raum einen fundierten Gesamtblick auf die Krise der EU bieten, den sie mit weitreichenden Reformvorschlägen verknüpfen.

Claus Offes bei Suhrkamp erschienenes Taschenbuch wendet sich an ein Publikum, das deutlich größer ist als die einer linken EU-Kritik üblicherweise Verdächtigen. Es ist nicht zuletzt für Leserinnen und Leser aus jenem politischen, akademischen und teilweise auch gewerkschaftlichen Mitte-Links-Milieu interessant, die die EU in ihrer jüngeren Entwicklung im Wesentlichen nach wie vor als die institutionelle Verkörperung jenes Friedens- und Fortschrittsprojekts betrachten, dessen historische Größe alle Schwächen überstrahlt, die diese Form der Integration heute im Einzelnen auch haben mag. Offe nimmt sich dieser Erwartungen und Hoffnungen mit großer Sympathie an, aber er unterzieht sie einem „ernüchternden Realitätstest“ (S. 93), der auch das viel gelobte Friedensprojekt einschließt, das ja Frieden nicht allein im Innern, sondern auch nach außen stiften sollte.

Hier können nur einige wenige Aspekte seiner Analyse hervorgehoben werden, die in den Befund mündet, dass die EU sich heute an einem Scheideweg befinde: „Entweder gelingt eine erhebliche Verbesserung ihrer institutionellen Struktur oder es kommt zu ihrem Zerfall“ (S.13). Offe macht zum einen (unter Bezug auf Fritz Scharpf) die Strategie einer „negativen Integration“ dafür verantwortlich, dass in den Mitgliedstaaten „Arbeitsmärkte und Sozialsysteme unter Liberalisierungsdruck gesetzt wurden“ (S. 96). Zum zweiten kritisiert er das - darauf aufbauende - „stabilitätsbesessene Währungsregime“ (S.35), das „aus sehr heterogenen Volkswirtschaften besteht, deren Unterschiede durch den Euro nur noch vertieft wurden“ (S. 40). Dass die Wurzeln dieser „Fehlkonstruktion“ (S. 127) tief reichen, beleuchtet er schließlich mit seiner Analyse des politischen Systems der EU als einem „eigentümlichen Nicht-Staat", dessen oberste politische Entscheidungsins$\tan z$ der Europäische Rat der Staats- und Regierungschefs ist, also ein intergouvernementales Gremium, dessen Mitglieder „,zwar in ihren jeweiligen Ländern gewählt worden sind, um die jeweilige Nation zu regieren, nicht aber die Europäische Union“ (S. 152ff.).

Damit lenkt Offe die Aufmerksamkeit auf das vieldiskutierte „Demokratiedefizit“. Diese „hässliche Seite des EU-Herrschaftssystems“ (S. 152) zu überwinden, ist in seinen Augen überaus schwierig, denn das, was zur Überwindung der Krise der EU erforderlich wäre, sei für „nationale Machterwerbsorganisationen“ den jeweiligen Wählerinnen und Wählern sehr schwer zu „verkaufen“ (S. 17). Die Beschreibung dieses Dilemmas, dem das Buch auch seinen Titel „Europa in der Falle“ verdankt, verleitet den Autor jedoch weder zu dem auch in linken Kreisen beliebter gewordenen Plädoyer für einen „Rückbau“ als vermeintlicher Alternative zu einer „Vertiefung“ der EU, noch zu einer indirekten Entschuldigung der nationalstaatlichen Eliten - zu allerletzt der deutschen, der er ein eigenes Kapitel widmet. Den in Deutschland dominierenden politischen und medialen Diskurs im Verlaufe der Euro-Krise (und insbesondere der fortdauernden Auseinandersetzung über Griechenland) bezeichnet er als „konsequent nationalistisch“, da er sich damit begnüge, ,andere darüber zu belehren, welche Fehler sie in der Vergangenheit besser nicht begangen hätten“, anstatt sich der „Mühe der Formulierung einer Strategie für die Eurozone insgesamt“ zu unterziehen (S. 127f.). Deutschland, so Offes Quintessenz, sollte nicht zur „Führung“ aufgefordert werden, sondern - auch und gerade im eigenen Interesse! - „zur Preisgabe von Teilen seiner wirtschaftlichen und politischen Vormachtstellung zugunsten der Stärkung von supranationaler europäischer Führungs- und Handlungskapazität" (S.142).

Dies unterstreicht, dass der Konflikt darüber, wie das beschriebene Dilemma überwunden und die EU aus der von den Regierungen ihrer Mitgliedsländer gebauten Falle befreit werden kann, in hohem Maße in Deutschland ausgetragen werden muss. Hier kommt Offes demokratischer Optimismus ins Spiel: „Politische Parteien (und ebenso die Medien)“ sollten nicht (vermeintlich) „gegebenen' und unabänderlichen Präferenzen der Wähler folgen“, sondern sich veranlasst sehen, „diese Präferenzen zu prägen ... und grenzüberschreitende Vertrauensbeziehungen zu schaffen“" (S. 17). Für möglich hält er eine solche Wende im Zusammenhang mit einem umfassenden - also sowohl die nationalstaatliche als auch die europäische Ebene einbeziehenden - politischen Großprojekt der „Umverteilung zwischen Staaten, Klassen und Generationen" (S. 166). Was manchen als zu gigantisch erscheinen mag, um wahr werden zu können, wird sich vielleicht - um es mit meinen eigenen Worten auszudrücken - früher oder später als die realistischste unter allen unrealistischen fortschrittlichen Alternativen zum Zusammenbruch der Eurozone und des Zerfalls der EU erweisen.

Ohne hier auf die - in dem Buch ohnehin nur kursorisch dargestellten - Einzelheiten dieses Projekts eingehen zu können, möchte ich den Grundgedanken hervorheben, der über viele linke Debatten hinausweist, die sich in der Möglichkeit oder Unmöglichkeit institutioneller Reformen der EU erschöpfen: Immer mehr wirtschaftliche und soziale Probleme in den Ländern der EU können „allein durch supranationales Handeln und Lastenteilung zwischen den Mitgliedstaaten bewältigt werden“; daher gebe es einen „Zusammenhang von inhaltlichen Problemen mit den Prozeduren der Willensbildung" (S. 167f.). Wenn dies berücksichtigt werde, könne ein Positivsummenspiel von „positiver Integration“ und „Input-Legitimation“, also Demokratisierung der EU in Gang gebracht werden.

Diese Überlegung zum Zusammenhang von Inhalt und institutionellen Prozeduren, also zum politischen Prozess einer Rettung der EU auf dem Wege ihrer demo- 
kratischen und sozialen Reform halte ich für so wichtig, dass der wenig ausgearbeitete Charakter der Reformprojekte im Einzelnen einstweilen zu verschmerzen ist.

Diese Lücke wird ausgefüllt durch die von einer Gruppe ausgewiesener EU-Expertinnen und -experten verfasste "Streitschrift für eine andere EU“. Ähnlich wie Offe kommt die von Busch et al. mit großer Detailkenntnis vorgelegte Analyse der mit dem Maastricht-Vertrag begründeten Wirtschafts- und Währungsunion zu dem Schluss, dass diese wegen der fehlenden gemeinsamen Wirtschafts- und Fiskalpolitik „die europäischen Staaten nicht zusammengeführt, sondern die Gräben zwischen den Staaten vertieft" habe (S.10). Die Autorinnen und Autoren betrachten sowohl die Euro-Krise als auch die im Zuge des Flüchtlingsdramas zutage getretene „Krise des Schengen-Systems“ als Ausdruck dafür, dass der neoliberal inspirierte Integrationsmodus an seine Grenzen gestoßen sei.

Auch Busch et al. setzen sich (mit wesentlich größerem Gewicht auf den ökonomischen Zusammenhängen als Claus Offe) kritisch mit der Idee auseinander, die wirtschaftlichen und politischen Fliehkräfte seien nur noch mit einem Ausstieg aus der „Fehlkonstruktion“ des Euro zu bewältigen. Dieser Teil der Streitschrift hat tatsächlich den gewünschten Streit ausgelöst, doch andere - mir noch wichtiger erscheinende Abschnitte - bis jetzt leider nur vereinzelt. Denn der Hauptteil des Plädoyers für ein „solidarisches Europa“ besteht in der Skizzierung von „sechs Säulen einer radikalen Euro-Reform“ (S.53ff.), nämlich der Ablösung der Austeritätspolitik durch expansive Fiskalpolitik und europäische Investitionsprogramme, der Schaffung einer „europäischen Ausgleichsunion“ zur Behebung der Leistungsbilanz-Ungleichgewichte, einer gemeinschaftlichen Schuldenpolitik, Wegen zu einer europäischen Sozialunion, einer Verschärfung der Finanzmarktregulierung und einer schlagkräftigeren europäischen Steuerpolitik sowie einer demokratisch legitimierten Europäischen Wirtschaftsregierung.

Die 30 Seiten, auf denen diese sechs Säulen vorgestellt werden, drängen sich als Basis einer kritischen EU-Reformdebatte geradezu auf. Zunächst deshalb, weil sie weit über die These hinausgehen, die gegenwärtigen Probleme seien allein durch wirtschaftspolitische Kurskorrekturen zu beheben. Nicht, dass derartige realpolitische Herangehensweisen von den Autorinnen und Autoren gering geschätzt würden - im Gegenteil: So wird am Beispiel des Umgangs mit der „Flüchtlingskrise“ gezeigt, welch zentrale Bedeutung die Schaffung eines neuartigen Fonds gewinnen könnte, mit dessen Hilfe Zuschüsse für Infrastrukturmaßnahmen insbesondere an aufnahmewillige Kommunen gegeben werden. Die strategische Bedeutung eines solchen Projekts bestünde in der Förderung einer bottom-up Herangehensweise in der europäischen Investitionspolitik, die in diesem spezifischen Kontext den besonderen Reiz hätte, wie die an dieser Stelle zitierte Gesine Schwan schreibt, „dass es den Nationalstaaten schwerer fällt, Flüchtlinge abzulehnen, wenn sich Gemeinden aus eigenem Interesse für die Aufnahme melden“ (S. 30). Doch im Rahmen des „sechs Säulen“-Programms unterstreichen derartige bereits im Rahmen der geltenden Verträge mögliche Aktionen den politischen Willen der dazu bereiten Mitgliedstaaten im Rahmen der sogenannten „verstärkten Zusammenarbeit“, zugleich aber auch die Notwendigkeit weiterreichender Reformen und können die Türen in diese Richtung öffnen.

Dass ein Konzept für derartige weiterreichende Reformen der Wirtschafts- und Währungsunion ausbuchstabiert wird, ist in meinen Augen das Hauptverdienst der Streitschrift. Gerade dies offenbart aber zugleich ihre wichtigste Schwäche. Denn je mehr sie - verdienstvoller Weise! - ins Kleingedruckte geht und nicht bei Alternativ-Vorschlägen stehen bleibt, an denen wahrlich, wie Offe anmerkt, kein Mangel besteht, desto deutlicher fallen Risiken und Nebenwirkungen ins Auge. Das vielleicht größte Risiko ist die ungewollte Schützenhilfe für ein autoritäres Durchregieren unter neoliberalen Vorzeichen, die mit der Einführung einer EU-Wirtschaftsregierung gegeben werden könnte, ohne dass durch Vertragsreformen der in der de-facto-Verfassung verankerte Vorrang wirtschaftlicher „Grundfreiheiten“vor sozialen und demokratischen Rechten gebrochen und die EU-Kommission eine wesentlich stärkere sowohl demokratische als auch finanzielle Basis bekäme. So betrachtet wäre eine Wirtschaftsregierung weniger als eine Säule unter mehreren zu verstehen, sondern eher als Ergebnis eines längeren grundlegenden Umbauprozesses.

In der Zusammenschau sind beide Veröffentlichungen auf eine zum Nachdenken anregende Weise komplementär. Einerseits könnte ein sachkundig ausgearbeitetes Reformprogramm wie das der Streitschrift zur Bereicherung der Überlegungen von Offe dienen. Andererseits würde das Weiterverfolgen und Konkretisieren (!) von Offes Gedanke, dass erst aus der Verzahnung von Inhalt und Prozeduren der Willensbildung neue politische Handlungsfähigkeit erwachsen kann, der Streitschrift noch größere Überzeugungskraft verleihen. Denn die demokratische und soziale Reform der EU ist, wenn überhaupt, nur als langwieriger und konfliktreicher Prozess vorstellbar, der von Solidarität im jeweils eigenen Interesse der nationalstaatlichen Akteure angetrieben wird. Denkanstöße, wie sie die beiden hier vorgestellten Veröffentlichungen geben, sind für die Gewinnung einer Handlungsfähigkeit von unten unverzichtbar.

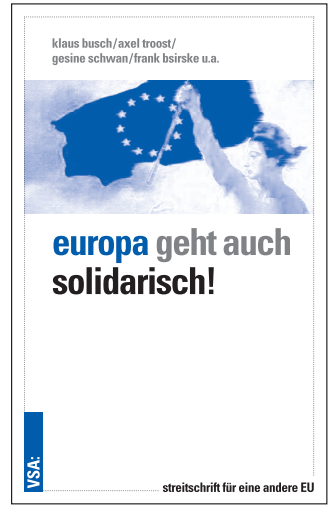

BUSCH, K. ET AL. EUROPA GEHT AUCH SOLIDARISCH! STREITSCHRIFT FÜR EINE ANDERE EUROPÄISCHE UNION

VSA Verlag Hamburg, 2016

ISBN 978-3-89 965-745-6 Seiten, $7,50 €$ 


\section{Abstracts}

WSI-MITTEILUNGEN 2/2019, SEITEN 87-95 GÜNTHER SCHMID

\section{Europa in Arbeit: Plädoyer für eine neue Vollbeschäftigung durch inklusives Wachstum}

Prominente Ökonomen und Sozialwissenschaftler sehen im Euro die zentrale Ursache für die gegenwärtige Krise Europas. Die politischen und ökonomischen Kosten einer Aufgabe des Euro wären jedoch riesig. Der Euro könnte sogar zum Dreh- und Angelpunkt eines Projekts des „Sozialen Europa“ werden, wenn dem gegenwärtigen Finanzkapitalismus eine realitätsgerechte Politik der neuen Vollbeschäftigung durch inklusives Wachstum entgegengesetzt würde. Dieser Beitrag bietet hierzu konkrete Reformvorstellungen, insbesondere im Zusammenhang mit atypischen Formen der Beschäftigung und der möglichen Ausgestaltung einer europäischen Arbeitsversicherung.

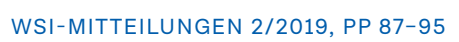

\section{Europe in work: A plea for new full employment through inclusive growth}

Prominent economists and social scientists consider the central cause for the current crisis in Europe to be the euro. The political and economic costs of giving up the euro, however, would be tremendous. The euro, could, in fact, be the key issue for a project of "Social Europe", if only the prevalent financial capitalism were to be countered by a realistic policy of new full employment through inclusive growth. This article provides concrete reform proposals in this direction, in particular related to non-standard forms of employment and the potential creation of a European employment insurance.
WSI-MITTEILUNGEN 2/2019, SEITEN 96-105 ANKE HASSEL, SOPHIA VON VERSCHUER

Ein europäischer Rechtsrahmen für Arbeitnehmerbeteiligung in transnationalen Unternehmen

In Anbetracht der Machtfülle transnationaler Unternehmen und der sozialen Auswirkungen zunehmender Globalisierung sind neue Formen der gesellschaftlichen Einbettung von Unternehmen nötig. Arbeitnehmerbeteiligung an strategischen Unternehmensentscheidungen ist ein wichtiges Instrument zur Korrektur unternehmerischer Entscheidungen und sie ist insbesondere ein Kernbestandteil des europäischen Sozialmodells. Die Arbeitnehmerbeteiligung bleibt jedoch derzeit unterentwickelt und den gegenwärtigen Herausforderungen nicht angemessen. Ein wichtiger Ansatz zu ihrer Stärkung basiert auf der Vergewisserung ihrer Funktionen in einem institutionell heterogenen Umfeld. Verbindliche Formen der Arbeitnehmerbeteiligung müssen eine aktive Rolle bei der Formulierung von Arbeitnehmerinteressen und in der Unternehmensaufsicht spielen. Im Europarecht gibt es durchaus positive Ansätze zur Verankerung von Beteiligungsrechten von Arbeitnehmerinnen und Arbeitnehmern, die jedoch durch weitere Integration des Gesellschaftsrechts unterminiert werden. Eine Lösung besteht in der Formulierung von Bereichsausnahmen in den Regelungen zum EU-Binnenmarkt und einer aktiveren politischen Gestaltung eines arbeitnehmerfreundlichen europäischen Gesellschaftsrechts.

WSI-MITTEILUNGEN 2/2019, PP 96-105 ANKE HASSEL, SOPHIA VON VERSCHUER

\section{A European legal framework for workers' voice in transnational companies}

Given the power of transnational corporations and the social impact of increasing globalisation, new forms of social embedding of companies are called for. Workers' voice in the form of employee participation in strategic business decisions is an important tool for monitoring 
business decisions and is a key component of the European social model. However, it is currently underdeveloped and inadequate to meet current challenges. An important approach to strengthening employee participation is based on ensuring their functions in an institutionally diverse environment. Binding forms of worker participation must play an active role in formulating workers' interests and in monitoring corporate behaviour. European law has a number of positive approaches to the anchoring of participation rights of employees such as the European Social Charter or the recent European Pillar of Social Rights. However, the European-level development of proposals for company law and decisions by the ECJ tend to undermine social standards. European solutions to these issues are the formulation of exceptions to the norms of the EU single market as well as new policy advances towards a more worker-friendly European company law.

WSI-MITTEILUNGEN 2/2019, SEITEN 106-114 HANS-WOLFGANG PLATZER, TORSTEN MÜLLER

\section{Die Europäischen Gewerkschafts- verbände: Zur Entwicklung ihrer} Machtressourcen und Funktionsprofile

Der Beitrag untersucht, wie sich im Verlauf der 2007/2008 einsetzenden Finanzmarkt- und Euro-Krise die Machtressourcen, Aufgaben und Politiken der Europäischen Gewerkschaftsverbände (EGV) verändert haben. Die Analyse zeigt, dass keines von zwei angesichts der Wucht der Krise denkbaren Extremszenarien eintrat: Weder kam es durch Re-Nationalisierungstendenzen zu einer Blockade der transnationalen gewerkschaftlichen Zusammenarbeit und einer Erosion der Handlungsfähigkeit der EGV; noch kam es aufgrund gesteigerter Solidaritätsanforderungen zu einer Vertiefung der transnationalen gewerkschaftlichen Zusammenarbeit unter dem Dach der EGV, sodass sich der heutige Entwicklungsstand der EGV im Vergleich zur Vorkrisenperiode als Status quo ante beschreiben lässt. Allerdings fördert die Empirie ein nach verschiedenen Handlungsfeldern differenziertes Bild gradueller Veränderungen im Zeit- verlauf zutage. Der Beitrag verdeutlicht auch, dass sich vor dem Hintergrund teils geschwächter nationaler Machtressourcen und einer tendenziell gewachsenen Heterogenität nationaler Gewerkschaftsinteressen die Formulierung gemeinsamer europäischer Handlungsansätze schwieriger gestaltet als in vorausgegangenen Entwicklungsphasen.

WSI-MITTEILUNGEN 2/2019, PP 106-114 HANS-WOLFGANG PLATZER, TORSTEN MÜLLER

The European trade union federations: the development of their power resources and roles

The article examines how the power resources, tasks and policies of the European trade union federations (ETUFs) changed in the course of the financial crisis which started in 2007/2008. The analysis illustrates that neither of the two conceivable extreme scenarios have materialised: re-nationalisation trends did not block transnational trade union cooperation or erode the ETUFs' capacity to act; nor did increased transnational solidarity requirements lead to intensified transnational cooperation under the umbrella of the ETUFs. What we can observe instead are only gradual changes occurring over time and varying by policy area; hence, compared to the pre-crisis period, the ETUFs' current state can be characterised as status quo ante overall. The article, however, also shows that partially weakened national power resources and a trend towards an increased heterogeneity of national trade union interests have made the formulation of joint European strategies even more difficult today than in the past.

WSI-MITTEILUNGEN 2/2019, SEITEN 115-124 WILLI KOLL, ANDREW WATT

\section{Konvergenz von Lohnentwicklung und Makropolitik für eine stabile Europäische Wirtschafts- und Währungsunion}

Die nationale Lohn- und Preisentwicklung beeinflusst wesentlich Binnennachfrage, preisliche Wettbewerbsfähigkeit und Leistungsbilanz. Für eine gleichgewichtige Entwicklung im Euroraum muss deshalb nicht nur im Aggregat, sondern in jedem Mitgliedstaat die binnenwirtschaftliche Preisentwicklung im Einklang mit den Lohnstückkosten stehen und beide Größen müssen dem Preisziel der Geldpolitik entsprechen. Von dieser „Goldenen Regel der Lohn- und Preisentwicklung" sind einige Mitgliedstaaten - dauerhaft und gravierend - nach oben und andere nach unten abgewichen. Das Ergebnis waren der Aufbau massiver Divergenzen in Binnendynamik, Leistungs- und Kapitalbilanz und ihre Entladung in einer schweren Wirtschaftskrise.

Löhne und Preise sind sowohl Treiber als auch Getriebene der gesamtwirtschaftlichen Entwicklung. Damit tragen sowohl die Sozialpartner als auch die Wirtschafts- und Fiskalpolitik Verantwortung für die Einhaltung der Regel. Daraus folgt die Notwendigkeit einer Kooperation zwischen diesen makroökonomischen Akteuren und deren Institutionalisierung. Hierzu wird ein Reformvorschlag dergestalt vorgestellt, dass Spitzenvertreter von Geld-, Fiskal- und Lohnpolitik einen zielführenden Dialog zur Einhaltung der Goldenen Regel führen und anschließend unter Wahrung ihrer Unabhängigkeit ihre Politik entsprechend ausrichten.

WSI-MITTEILUNGEN 2/2019, PP 115-124 WILLI KOLL, ANDREW WATT

\section{Convergence of wage development and macroeconomic policy for a stable Economic and Monetary Union}

National wage and price developments are decisive for domestic demand, competitiveness and the current account. Balanced growth in the Euro area requires that, not only in the aggregate but in each member state, inflation is commensurate with nominal unit labour cost developments and both variables with the inflation target of the European Central Bank. Yet some member states deviated, in both directions and persistently, from this "Golden Rule of wages and prices". This led to a massive build-up of imbalances regarding domestic demand, current and capital accounts, whose tensions were only resolved via a deep economic and financial crisis. 
Wages and prices are both drivers of, and are driven by, macroeconomic developments. Thus both the social partners and fiscal and monetary policy makers bear responsibility for adhering to the Golden Rule. This requires cooperation between the macroeconomic policy actors which must be appropriately institutionalised. The authors present a reform proposal that would bring together representatives of monetary and fiscal policy and the social partners in a formal dialogue tasked with respecting the Golden Rule, tailoring their policies towards achieving it while maintaining their autonomy.

WSI-MITTEILUNGEN 2/2019, SEITEN 125-132 KLAUS BUSCH

\section{Rechtspopulismus in der EU - Bedrohung für den Integrationsprozess}

Für den Anstieg des Rechtspopulismus in wichtigen EU-Staaten (Italien, Frankreich, Österreich, Niederlande, Deutschland) sind vor allem fünf Einflussgrößen von besonderer Relevanz: ökonomische Probleme (Arbeitslosigkeit), die soziale Ungleichheit, die politische Stabilität/ Instabilität des Staates, die Migrations- und Flüchtlingsfrage sowie ein historisch-kultureller Faktor. Der Machtzuwachs des Rechtspopulismus hat in den betreffenden Mitgliedstaaten und in der EU die politische Instabilität verstärkt. Der Prozess der wachsenden Re-Nationalisierung verhindert in der EU die Überwindung von zentralen Integrationskonflikten. Dies gilt für einen obligatorischen Verteilungsmechanismus in der Flüchtlingspolitik und die Einführung einer makroökonomischen Stabilisierungsfunktion zur Bekämpfung von Krisen in der Eurozone. Zusammen mit dem Brexit und dem Abbau von Demokratie und Rechtsstaat in wichtigen Staaten Osteuropas gefährdet dies den Integrationsprozess.

WSI-MITTEILUNGEN 2/2019, PP 125-132 KLAUS BUSCH

\section{Right-wing populisms in the EU - threats to the integration process}

There are mainly five variables which explain the growing power of right-wing populism in key EU member states (Italy, France, Austria, the Netherlands, Germany): economic problems (unemployment), social inequality, political instability, the European refugee crisis and a historical-cultural factor. Right-wing populism is responsible for increasing political instability in the member states and the EU. The growing renationalisation is an obstacle to overcoming difficulties in significant integration conflicts. At the moment there is no solution for the redistribution of refugees between EU member states and for the creation of a European fiscal budget to fight economic crises in the EU. In combination with Brexit and the weakening of democracy and the rule of law in key Eastern European member states, the process of integration is being endangered.

WSI-MITTEILUNGEN 2/2019, SEITEN 133-141 THORSTEN SCHULTEN, MALTE LÜBKER

\section{WSI-Mindestlohnbericht 2019:}

\section{Zeit für kräftige Lohnzuwächse}

und eine europäische Mindestlohnpolitik

Die Rolle von Mindestlöhnen als Instrument zur Schaffung existenzsichernder Löhne ist in den letzten Jahren zunehmend in den Fokus der politischen Debatte gerückt. Vor diesem Hintergrund analysiert der WSI-Mindestlohnbericht 2019 die aktuelle Entwicklung der Mindestlöhne in den 22 Ländern der Europäischen Union, die eine gesetzliche Lohnuntergrenze haben, und darüber hinaus in 15 weiteren EU-Anrainerstaaten und wichtigen Volkswirtschaften in Übersee. Im Ergebnis zeigt sich, dass sich die Dynamik der Vorjahre fortsetzt und die Mindestlöhne in der EU zum Beginn des Jahre 2019 im Median um 4,8\% gestiegen sind. Getragen wird diese Entwicklung insbesondere von der anhaltenden Aufholbewegung der osteuropäischen Mitgliedstaaten, aber auch von einer substanziellen Anhebung in Spanien. Inzwischen erreichen die Mindestlöhne in der EU ein Niveau von gut $50 \%$ der Medianlöhne, womit sie jedoch weiterhin deutlich unter der Niedriglohnschwelle liegen. Eine koordinierte, strukturelle Anhebung der untersten Löhne ist dabei eine wichtige Zielsetzung für eine Europäische Mindestlohnpolitik.

WSI-MITTEILUNGEN 2/2019, PP 133-141 THORSTEN SCHULTEN, MALTE LÜBKER

\section{WSI Minimum Wage Report 2019:}

The time has come

for substantial wage gains

and a European minimum wage policy

Over the past few years, the potential of minimum wages as an instrument towards safeguarding adequate living wages has been increasingly recognised in the political discourse. Against this backdrop, the WSI Minimum Wage Report 2019 tracks current developments in all 22 EU Member States that set a statutory minimum wage, as well in 15 neighbouring countries in the EU and beyond. The report shows that the dynamic growth of minimum wages observed in the past extends into 2019, leading to a median increase of 4.8 per cent in the European Union. The trend is driven by further catch-up growth in Eastern Europe, and also by a substantial minimum wage adjustment in Spain. Averaging across the EU, minimum wages are now equivalent to just over 50 per cent of median wages. However, they still fall far short of the conventional low-pay threshold. A further increase is therefore one of the major objectives of a coordinated European minimum wage policy.
ABSTRACTS ZU DEN BEITRÄGEN IN DEN RUBRIKEN AUS DER PRAXIS UND DEBATTE UNTER WWW.WSI-MITTEILUNGEN.DE 


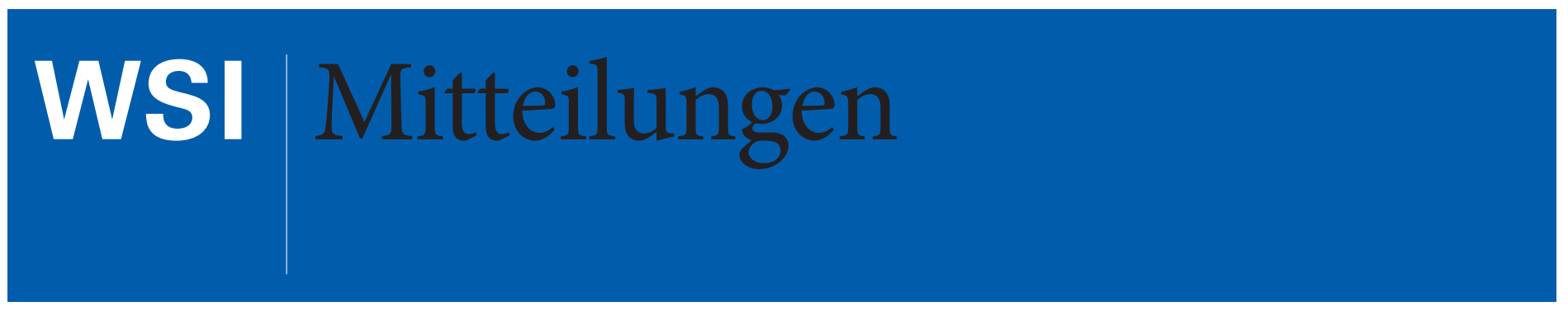

\title{
Schwerpunkthefte 2019
}

1|2019 Nachhaltige Arbeit: machtpolitische Blockaden und Transformationspotenziale

3|2019 Rechtspopulismus in der Arbeitswelt - eine Herausforderung für Gewerkschaften

5|2019 Umgang mit gesundheitlichen Beeinträchtigungen im Betrieb

\section{Schwerpunkthefte 2018}

\author{
1|2018 Altern im Betrieb \\ 3|2018 Industrie 4.0 konkret - Ungleichzeitige Entwicklungen, arbeitspolitische Einordnungen \\ 5|2018 Ungleichheitsdynamiken und Ungleichheitspolitiken
}

\section{Das Abonnement}

Jahresabonnement 2019*

Einzelabonnement Inland inkl. Onlinezugang (sechs Ausgaben Print inkl. Einzelplatzzugang) . . . . . . . . . . . . . . . . 94,80€ Einzelabonnement Ausland inkl. Onlinezugang (sechs Ausgaben Print inkl. Einzelplatzzugang) . . . . . . . . . . . . . . . 99,60€ Studentenabonnement In- und Ausland inkl. Onlinezugang (sechs Ausgaben Print inkl. Einzelplatznutzung) . . . . . . . . . 49,80€ Institutionsabonnement In- und Ausland inkl. Onlinezugang (sechs Ausgaben Print

\section{Miniabonnement**}

inkl. Onlinezugang über IP-Adresse für einen Standort) . . . . . . . 158,00€

3 Ausgaben der WSI-Mitteilungen im Miniabonnement Print, inkI. Vertriebskostenanteil

* Kündigungsfrist: drei Monate zum Kalenderjahresende

Preise inkl. MwSt., zzgl. Vertriebskostenanteil (Inland 24,00€ + Direktbeorderungsgebühr 2,15€) . . . . . . . . . . . . . . . . . . . 26,15€

(Ausland 45,00€ + Direktbeorderungsgebühr 2,15€) . . . . . . . . . . . . . . . . . . 47,15€

** Ich erhalte die Zeitschrift im regulären Abonnement, wenn ich nicht 1 Woche nach Erhalt des letzten Miniabo-Heftes abbestelle.

\section{Die Bestellung}

Ja, ich bestelle ein Abonnement der WSI-Mitteilungen als Einzelabonnement Inland

Ja, ich bestelle ein Abonnement der WSI-Mitteilungen als Einzelabonnement Ausland

Ja, ich bestelle ein Studentenabonnement der WSI-Mitteilungen

Ja, ich bestelle ein Institutionenabonnement der WSI-Mitteilungen

Ja, ich teste die WSI-Mitteilungen im Miniabonnement Ich erhalte dazu zwei Ausgaben der WSI-Mitteilungen in Print. Wenn ich innerhalb von 7 Tagen nach Erhalt der 2. Ausgabe keine gegenteilige Mitteilung mache, möchte ich die WSI-Mitteilungen regelmäßig beziehen.

Ja, ich bestelle oben angekreuzte Schwerpunkthefte

\section{Der Adressat}

Infotelefon: (0 72 21) 2104-39

Bestell-Fax: (0 72 21) 2104-1139

E-Mail: abo@nomos.de

Online Bestellmöglichkeit:

www.nomos-shop.de/30294

\section{Postadresse:}

Nomos Verlagsgesellschaft $\mathrm{mbH} \& \mathrm{Co}$. KG

Zeitschriftenvertrieb

Waldseestraße 3-5

76530 Baden-Baden

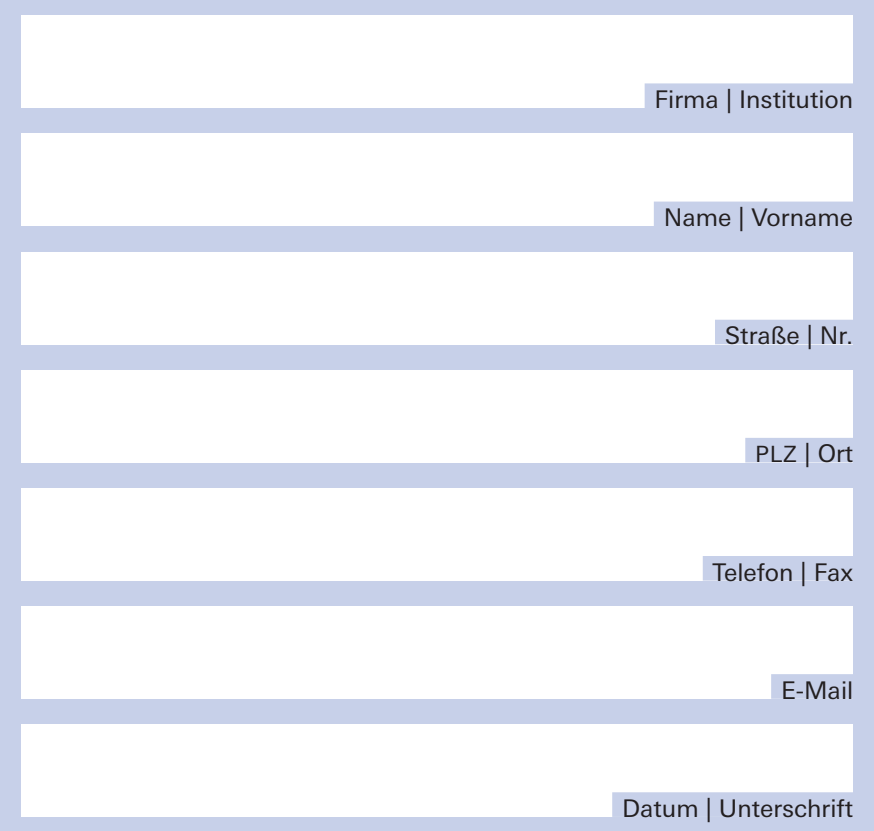

Ihre Vertrauensgarantie: Sie haben das Recht, diese Bestellung innerhalb von 14 Tagen bei der umseitigen Bestelladresse schriftlich zu widerrufen. Zur Wahrung der Frist genügt die rechtzeitige Absendung des Widerrufs. 
Die WSI-MITTEILUNGEN sind eine referierte wissenschaftliche Zeitschrift im Themenspektrum „Arbeit-Wirtschaft-Soziales“. Ihr Anliegen ist wissenschaftlicher Erkenntnisgewinn und der Transfer gesellschaftsrelevanter Wissenschaftsbefunde in die politische und gewerkschaftliche Praxis. Auf einen ansprechenden Sprachstil legen wir großen Wert. Namentlich gekennzeichnete Artikel geben die Meinung des Autors/der Autorin wieder, nicht unbedingt die der Herausgeberin und der Redaktion.

MANUSKRIPTANGEBote für die Rubriken Aufsätze, Forschung aktuell, Aus der Praxis, Debatte sowie Buchbesprechungen werden als Word-Dateien erbeten an die Redaktion: gudrun-linne@boeckler.de.

Das eingereichte Manuskript darf im deutschsprachigen Raum weder veröffentlicht sein noch einer anderen Zeitschrift gleichzeitig angeboten werden. Auch sind Originalbeiträge erwünscht. Die Zeitschrift sowie alle in ihr enthaltenen einzelnen Beiträge und Abbildungen sind urheberrechtlich geschützt. Jede Verwertung, die nicht ausdrücklich vom Urheberrechtsgesetz zugelassen ist, bedarf der vorherigen Zustimmung des Nomos Verlags. Mit der Annahme zur Veröffentlichung überträgt der Autor dem Verlag das ausschließliche Verlagsrecht für die Zeit bis zum Ablauf des Urheberrechts. Eingeschlossen sind insbesondere auch das Recht zur Herstellung elektronischer Versionen und zur Einspeicherung in Datenbanken sowie das Recht zu deren Vervielfältigung und Verbreitung online oder offline ohne zusätzliche Vergütung. Nach Ablauf eines Jahres kann der Autor anderen Verlagen eine einfache Abdruckgenehmigung erteilen; das Recht an der elektronischen Version verbleibt beim Verlag.

AUFSÄTZE sind analytische Beiträge zu einem praxisrelevanten Thema mit theoretischem wie auch empirischem Bezug (ca.40 ooo Zeichen inklusive Leerzeichen). Sie werden zweifach doppelt-blind begutachtet (Peer Review). Das Ergebnis wird üblicherweise binnen zwei Monaten nach Einreichung des Manuskripts mitgeteilt. Die Entscheidung über die Veröffentlichung in allen weiteren Rubriken liegt bei der Redaktion.

FORSCHUNG AKTUELL präsentiert zeitnah Befunde aus der empirischen Forschung, aus Datenanalysen oder gibt unter einer praxisrelevanten Fragestellung einen Überblick über den Forschungsstand (ca. 35 ooo Zeichen inklusive Leerzeichen).

In AUS DER PRAXIS werden aktuelle, praxisrelevante Entwicklungen dargestellt und kritisch kommentiert (max. 20 ooo Zeichen inklusive Leerzeichen).

DEBATTE bietet ein Forum für zugespitzte Diskussionsimpulse zu kontroversen wissenschaftlichen und/oder politischen Themen (max. 13000 Zeichen inklusive Leerzeichen).

BUCHBESPRECHUNGEN stellen Neuerscheinungen aus den Sozial- und Wirtschaftswissenschaften vor, die für einen breiten Leserkreis von Interesse sind (max.1150o Zeichen inklusive Leerzeichen). Für alle Textbeiträge gilt die neue deutsche Rechtschreibung gemäß den Empfehlungen des Dudens. Die detaillierten Redaktionshinweise finden Sie unter: www.boeckler.de/WSI-Mitteilungen. Wir bitten darum, diese bei der Texterstellung zu berücksichtigen.

www.boeckler.de/wsi-mitteilungen_45196.htm 